\title{
Mentale Gehalte und Erweiterter Geist - Warum das Argument der Nichtabgeleitetheit scheitert
}

\author{
Fabian Hundertmark \\ fhundertmark@uni-bielefeld.de \\ Universität Bielefeld | Abteilung Philosophie \\ Gebäude X, A4-241 | Postfach 10013 | 33501 Bielefeld
}

\begin{abstract}
Zusammenfassung
Der These des erweiterten Geistes zufolge befinden sich manche mentalen Repräsentationen außerhalb der körperlichen Grenzen der Wesen, zu denen sie gehören. Einer der stärksten Einwände gegen diese These stellt das Argument der Nichtabgeleitetheit von Frederick Adams, Ken Aizawa und Jerry Fodor dar. Dieses Argument setzt voraus, dass genuine mentale Repräsentationen nichtabgeleitete Gehalte haben - ihre semantischen Eigenschaften sind also nicht durch Absichten, Wünsche oder Konventionen konstituiert. Repräsentationen mit nichtabgeleitetem Gehalt finden sich jedoch, so das Argument weiter, nur innerhalb der körperlichen Grenzen mentaler Wesen. Ich werde dafür argumentieren, dass das Argument der Nichtabgeleitetheit scheitert, da es insbesondere bei Tieren externe Repräsentationen gibt, deren Gehalt nichtabgeleitet ist. Dies folgt jedenfalls aus der aussichtsreichsten Theorie nichtabgeleiteter Repräsentationen, der Teleosemantik, und es gibt gute Gründe anzunehmen, dass auch andere naturalistische Gehaltstheorien dieselbe Implikation haben.
\end{abstract}


Repräsentationen sind Träger semantischen Gehalts.11 Die These des erweiterten Geistes ist eine These über die Lokalisierung unserer mentalen Repräsentationen. Dieser These zufolge befinden sich manche unserer Überzeugungen, Wünsche und Absichten in unserer Umwelt: Wenn etwa der an Alzheimer erkrankte Otto ein Notizbuch verwendet, um durch das kontinuierliche Hinzufügen, Revidieren und Konsultieren von Einträgen die Konsequenzen seiner Krankheit abzumildern, so handelt es sich bei den Einträgen in seinem Notizbuch um seine Überzeugungen, Wünsche und Absichten. Schreibt er etwa in sein Notizbuch "The Museum of Modern Art is on 53rd Street", so handelt es sich bei diesem Eintrag nicht weniger um die Überzeugung, dass sich das Museum of Modern Art in der dreiundfünfzigsten Straße befindet, als bei Ingas neuronalem Zustand mit demselben Gehalt (vgl. Clark und Chalmers 1998, 12 - 16).

Einer der stärksten Einwände gegen die These des erweiterten Geistes ist das Argument der Nichtabgeleitetheit von Jerry Fodor (2008), Frederick Adams und Ken Aizawa (2001, 2005, 2008, 2010). Dieses Argument setzt voraus, dass genuine mentale Repräsentationen nichtabgeleitete Gehalte haben. Das heißt, dass eine Repräsentation nur dann eine mentale Repräsentation ist, wenn ihre semantischen Eigenschaften grob gesprochen nicht durch Absichten, Wünsche oder Konventionen konstituiert sind. Mentale Repräsentationen haben ihren Gehalt also nicht deshalb, weil sie mit bestimmten Absichten hervorgebracht werden, weil für sie bestimmte Konventionen gelten oder weil sie auf bestimmte Art und Weise interpretiert werden. Repräsentationen mit nichtabgeleitetem Gehalt finden sich jedoch, so Adams, Aizawa und Fodor weiter, nur innerhalb der körperlichen Grenzen mentaler Wesen. Folglich gibt es keine externen mentalen Repräsentationen.

Ich werde dafür argumentieren, dass das Argument der Nichtabgeleitetheit scheitert. Grund hierfür ist jedoch nicht die Falschheit der Annahme, dass nichtabgeleiteter Gehalt eine notwendige Bedingung für mentale Zustände sei. Diese Prämisse besitzt tatsächlich einiges an Plausibilität. Problematisch ist vielmehr die empirische Annahme, dass sich außerhalb zentraler Nervensysteme nur Repräsentationen mit abgeleitetem Gehalt befinden.

Diese Annahme lässt sich nicht unabhängig von einer Theorie des Gehalts überprüfen. Die plausibelste Theorie dieser Art ist die Teleosemantik. Etwas vereinfacht gesagt, hat eine Repräsentation der Teleosemantik zufolge den Gehalt, dass $p$, wenn sie die natürliche

\footnotetext{
${ }^{1}$ In meiner Terminologie sind Repräsentationen (z. B. Wünsche, Überzeugungen und Absichten) physische Vehikel, welche die Eigenschaft haben, semantischen Gehalt zu tragen. Die Gehalte sind dabei keine Komponenten von Repräsentationen, obwohl eine Repräsentation nur aufgrund ihres semantischen Gehalts als Repräsentation zählt. In gleicher Weise ist eine Eigenschaft, etwas verursacht zu haben, keine Komponente einer Ursache, obwohl die Ursache nur aufgrund dieser Eigenschaft als Ursache zählt.
} 
Funktion hat, anzuzeigen, dass $p$ der Fall ist. Funktionen dieser Art spielen eine große Rolle in der Medizin und Biologie. Zum Beispiel, wenn wir Organen Fehlfunktionen zuschreiben. Ich werde anhand von Beispielen zeigen, dass natürliche Anzeigefunktionen und somit nichtabgeleitete Gehalte nicht auf Organe und insbesondere nicht auf das Gehirn beschränkt sind.

In meiner Argumentation werde ich wie folgt vorgehen: Im Abschnitt 1 werde ich zunächst den Begriff der Repräsentation, des Gehalts und der Erfüllungsstandards einführen. In Abschnitt 2 werde ich die These des erweiterten Geistes darstellen und gegen andere Thesen abgrenzen. Anschließend werde ich in den Abschnitten 3 und 4 das Argument der Nichtabgeleitetheit darstellen und ein adäquates Verständnis von nichtabgeleitetem Gehalt herausarbeiten. In Abschnitt 5 werde ich dafür argumentieren, dass es bei der Unterscheidung zwischen abgeleitetem und nichtabgeleitetem Gehalt um die Quelle der repräsentationalen Erfüllungsstandards geht und dass Repräsentationen mit nichtabgeleitetem Gehalt ihre Erfüllungsstandards plausiblerweise durch natürliche Funktionen erhalten. Dann werde ich in Abschnitt 6 zeigen, dass sich die Art von Funktion, die für nichtabgeleiteten Gehalt notwendig ist, nicht auf die Körper von Tieren beschränkt und dass uns das Argument der Nichtabgeleitetheit somit keinen Grund gibt, die These des erweiterten Geistes zu bestreiten. Abschließend werde ich in Abschnitt 7 kurz dafür argumentieren, dass Fodors eigene Theorie nichtabgeleiteten Gehalts für das Argument der Nichtabgeleitetheit kein besseres Ergebnis liefert als die Teleosemantik.

\section{Repräsentationen und ihre Erfüllungsstandards}

Grice (1957, S. 377f.) unterscheidet Sätze wie „Diese Flecken bedeuten Masern“, in denen es um natürliche Bedeutung (Bedeutung ${ }_{n}$ ) geht, von Sätzen wie „Das dreifache Klingeln der Glocke bedeutet, dass der Bus voll ist", in denen von nicht-natürlicher Bedeutung $\left(\right.$ Bedeutung $_{n n}$ ) die Rede ist. Gegenstände, Zustände, Strukturen oder Ereignisse (kurz: Vehikel) die Bedeutung ${ }_{n n}$ haben, sind Repräsentationen. Das, was eine Repräsentation bedeutet $_{n n}$ oder repräsentiert, ist ihr semantischer Gehalt.

Grice weist darauf hin, dass der Satz „Diese Flecken bedeuten Masern“ nur dann wahr sein kann, wenn der Patient tatsächlich Masern hat. $R$ kann offenbar nur dann $G$ bedeuten $_{n}$, wenn $G$ vorliegt. Dies gilt für nicht-natürliche Bedeutung nicht. Das dreifache Klingeln der Glocke kann auch dann bedeuten $_{n n}$, dass der Bus voll ist, wenn der Bus tatsächlich nicht voll ist. Es können also nicht-bestehende Sachverhalte repräsentiert werden. Repräsenta- 
tionen mit Wahrheits- oder Erfüllungsbedingungen können wahr oder falsch bzw. erfüllt oder nicht-erfüllt sein, je nachdem, ob der repräsentierte Sachverhalt besteht oder nicht. Anders ausgedrückt: Die Eigenschaft, Bedeutung ${ }_{n}$ zu haben, ist faktiv, die Eigenschaft,

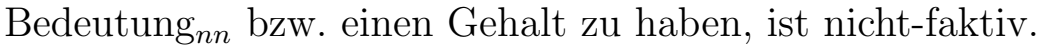

Ein weiterer Unterschied zu natürlicher Bedeutung ist die Spezifität nicht-natürlicher Bedeutung. So gilt für alle Flecken, die Masern bedeuten ${ }_{n}$, dass sie Krankheit bedeuten ${ }_{n}$ und auch, dass sie bedeuten ${ }_{n}$, dass sich die Person, die die Flecken hat, per Tröpfcheninfektion bei einem anderen Menschen mit dem Masernvirus infiziert hat. Wenn y immer

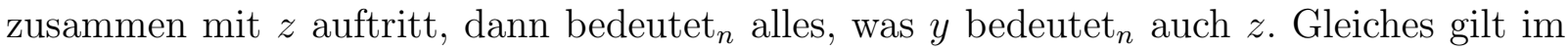
Fall nicht-natürlicher Bedeutung nicht. Man kann durchaus mit einem Satz meinen, dass der Patient Masern hat, ohne zu meinen, dass der Patient krank ist. Ebenso kann man meinen, dass der Patient Masern hat, ohne zu meinen, dass er sich bei einem anderen Menschen per Tröpfcheninfektion mit dem Masernvirus infiziert hat.

Die Spezifität repräsentationalen Gehalts erschöpft sich jedoch nicht darin, dass etwas $x$ repräsentieren kann, ohne $y$ zu repräsentieren, auch wenn $x$ und $y$ immer zusammen auftreten. Dies gilt schließlich auch für einfache Kausalbeziehungen. Der Sturm wird etwa von schnell fallendem Luftdruck verursacht und nicht vom schnell fallenden Barometerstand, auch wenn Barometerstand und Luftdruck immer gleichzeitig fallen. Repräsentationale Beziehungen sind nicht nur spezifischer als Korrelationsbeziehungen, sondern auch als Kausalbeziehungen. Etwas kann $x$ repräsentieren, ohne $y$ zu repräsentieren, auch wenn $x y$ verursacht oder umgekehrt. So kann man etwa behaupten, dass der Patient Masern hat, ohne zu behaupten, dass der Patient sich bei seiner Schwester angesteckt hat oder dass der Patient die nächsten Tage nicht zur Arbeit gehen wird. Auch wenn es sich bei der Ansteckung des Patienten um eine Ursache und der Abwesenheit des Patienten bei der Arbeit um eine Wirkung der Masern handelt.

Die Eigenschaft, einen Gehalt bzw. eine Bedeutung ${ }_{n n}$ zu haben, geht also, im Gegensatz zur Eigenschaft, Bedeutung ${ }_{n}$ zu haben, mit Erfüllungsstandards einher ${ }^{2}$ Diese Erfüllungsstandards zeichnen sich dadurch aus, dass sie zum einen nicht-faktiv und zum anderen spezifischer als Korrelations- und Kausalbeziehungen sind.

\footnotetext{
${ }^{2}$ Häufig wird von "normativen" oder "quasi-normativen" Eigenschaften repräsentationalen Gehalts gesprochen. Dieser Bezug auf Normen ist jedoch gleich doppelt problematisch, sodass ich lieber von "Erfüllungsstandards" sprechen möchte. Zum einen meine ich, dass sich die hier gemeinten Erfüllungsstandards anders als wirkliche Normativität im Prinzip problemlos aus rein deskriptiven Aussagen ableiten lassen. Zum anderen sollten wir die hier gemeinten Erfüllungsstandards von der Normativität unterscheiden, auf die wir etwa Bezug nehmen, wenn wir Personen ihre falschen Überzeugungen oder Aussagen vorwerfen.
} 
Wünsche, Überzeugungen und Absichten unterliegen Erfüllungsstandards im relevanten Sinne. Ich kann den Wunsch oder die Überzeugung haben, dass die Sonne scheint, ohne dass tatsächlich die Sonne scheint. Ich kann wünschen oder glauben, dass ein Tiger vor mir ist, ohne zu wünschen oder zu glauben, dass sich ein Tier der Art Panthera tigris vor mir befindet und ich kann den Wunsch oder die Absicht haben, zwei Flaschen Wodka zu leeren, ohne den Wunsch oder die Absicht zu haben, ins Koma zu fallen, auch wenn das Koma eine unvermeidbare Folge eines solchen Alkoholkonsums wäre. Bei Wünschen, Überzeugungen und Absichten handelt es sich also um klare Fälle mentaler Repräsentationen.

\section{Erweiterter Geist}

In der Philosophie des Geistes der zweiten Hälfte des letztes Jahrhunderts wurde zumeist davon ausgegangen, dass mentale Repräsentationen bestimmte Zustände des Gehirns kognitiver Wesen seien. Doch spätestens seit Clark und Chalmers (1998) ist diese Annahme umstritten. Betrachten wir das bekannte Beispiel von Otto und Inga:

First, consider a normal case of belief embedded in memory. Inga hears from a friend that there is an exhibition at the Museum of Modern Art, and decides to go see it. She thinks for a moment and recalls that the museum is on 53rd Street, so she walks to $53 \mathrm{rd}$ Street and goes into the museum. It seems clear that Inga believes that the museum is on 53rd Street, and that she believed this even before she consulted her memory. It was not previously an occurrent belief, but then neither are most of our beliefs. Rather, the belief was sitting somewhere in memory, waiting to be accessed. Otto suffers from Alzheimer's disease, and like many Alzheimer's patients, he relies on information in the environment to help structure his life. In particular, Otto carries a notebook around with him everywhere he goes. When he learns new information, he writes it down in his notebook. When he needs some old information, he looks it up. For Otto, his notebook plays the role usually played by a biological memory. Today, Otto hears about the exhibition at the Museum of Modern Art, and decides to go see it. He consults the notebook, which says that the museum is on 53rd Street, so he walks to 53rd Street and goes into the museum.

Clearly, Otto walked to 53rd Street because he wanted to go to the museum and he believed the museum was on 53rd Street. And just as Inga had her belief even before she consulted her memory, it seems reasonable to say that Otto believed the museum was on 53rd Street even before consulting his notebook. For in relevant respects the 
cases are entirely analogous: the notebook plays for Otto the same role that memory plays for Inga. The information in the notebook functions just like the information that' constitutes an ordinary non-occurrent belief; it just happens that this information inheres in a physical state located beyond the skin. (Clark und Chalmers 1998 $12-13$, meine Hervorhebung)

Vertreter der These des erweiterten Geistes behaupten, dass unsere mentalen Repräsentationen nicht nur in unseren Köpfen, sondern auch in unserer Umwelt zu finden sind. Manche unserer Überzeugungen, Wünsche und Absichten befinden sich demnach nicht in unseren Gehirnen, sondern in den Speichern unserer Smartphones oder in unseren Notizblöcken. Geknotete Taschentücher, zählende Finger und Tätowierungen können echte Überzeugungen darstellen. Die These des erweiterten Geistes lässt sich wie folgt verstehen:

\section{These des erweiterten Geistes}

Es gibt externe mentale Repräsentationen ${ }^{3}$

Wobei externe Repräsentationen wie folgt definiert sind:

\footnotetext{
${ }^{3}$ Zwei Anmerkungen hierzu: Zum einen ist hier streng genommen ein Spezialfall der These des erweiterten Geistes dargestellt, da von mentalen Repräsentationen und nicht etwa von mentalen Prozessen oder kognitiven Systemen die Rede ist. Insbesondere Antirepräsentationalisten und Enaktivisten, die auf der einen Seite die Rolle von Repräsentationen in kognitiven Systemen herunterspielen oder gar ganz bestreiten und auf der anderen Seite die Rolle der Interaktion mit der Umwelt für eine zentrale Eigenschaft kognitiver Systeme halten, werden mit der hier dargestellten These nicht einverstanden sein. Dennoch können sie eine Form der These des erweiterten Geistes vertreten, der zufolge sich mentale Prozesse oder kognitive Systeme über die Körper der Wesen, zu denen sie gehören, hinaus in die Umwelt erstrecken. Da es sich bei den von mir besprochenen Autoren aber um Anhänger eines Repräsentationalismus handelt, spricht in diesem Kontext nichts dagegen, die These des erweiterten Geistes entsprechend zu verstehen.

Zum anderen wollen die meisten Vertreter der These des erweiterten Geistes nicht nur behaupten, dass externe mentale Repräsentationen existieren, sondern dass es sich bei diesen um verbreitete Phänomene handelt, die eine große Rolle in den Wissenschaften spielen sollten, die sich mit dem Geist beschäftigen. Da das von mir besprochene Argument der Nichtabgeleitetheit aber die bloße Existenz externer mentaler Repräsentationen bestreitet, reicht es für meine Zwecke aus, die These des erweiterten Geistes als Existenzbehauptung zu lesen.
} 


\section{Externe Repräsentationen}

Eine Repräsentation $R$ ist extern gdw.

1. $R$ zu dem Wesen $C$ gehörtt und

2. $R$ sich teilweise oder komplett außerhalb der körperlichen Grenzen von $C$ befindet.

Die These des erweiterten Geistes ist dabei explizit so zu verstehen, dass es bei ihr um die Lokalisierung mentaler Repräsentationen im Sinne von Vehikeln, also den Trägern repräsentationaler Eigenschaften geht. Sie ist somit von einem semantischen Externalismus zu unterscheiden, der die Eigenschaft, etwas bestimmtes zu repräsentieren, als Eigenschaft auffasst, die nicht nur durch körperinterne Tatsachen konstituiert ist. Während Hilary Putnam einen solchen semantischen Externalismus in dem Slogan „,meanings“ just ain’t in the head!"(Putnam 1979, S. 227) zum Ausdruck bringt, lässt sich die These des erweiterten Geistes wie folgt ausdrücken: "The material vehicles of some mental representations just ain't in the head!"

Um eine solche These zu begründen, reicht es nicht aus, wie Clark und Chalmers es tun, einen wechselseitigen kausalen Einfluss zwischen Geist und Umwelt nachzuweisen. So stellen etwa soziale Interaktionen zum Teil sehr intensive gegenseitige Beeinflussungen kognitiver Systeme dar, ohne dass sich die beteiligten Systeme nur aufgrund dieser Beeinflussung zu einem neuen kognitiven System vereinen würden (auch wenn dadurch eventuell soziale Systeme entstehen). Ein gerechtfertigter Schluss von kausaler Kopplung auf Konstitution ist, wie auch Adams und Aizawa (2001, 2008 und Aizawa 2010) plausibel gemacht haben, nicht möglich.

Auch behauptet die These des erweiterten Geistes nicht nur, dass wir zur Erklärung unseres Geistes externe Faktoren mit einbeziehen müssen. Denn selbst die Gegner dieser These bestreiten nicht, dass unser Geist in den allermeisten Fällen bestimmte Umweltbedingungen benötigt, die als Input dienen, während sehr häufig Handlungen die Outputs geistiger Prozesse darstellen. Sowohl nichtgeistige Inputs als auch nichtgeistige Outputs gehören selbstverständlich zur Erforschung und Erklärung geistiger Fähigkeiten dazu. Kognition stellt hier keine Ausnahme dar. Ebenso wie es für die Erforschung und Erklärung

\footnotetext{
${ }^{4}$ Natürlich darf es dafür, dass $R$ zu dem Wesen $C$ gehört, nicht notwendig sein, dass sich $R$ räumlich in $C$ befindet. Eine ähnliche Feststellung macht Rowlands (2009, S. 15ff.; 2010, S. 135 - 162) in Bezug auf kognitive Prozesse, deren Zugehörigkeit ihm zufolge von der richtigen Integration in das psychologische Leben des Wesens abhängt. Da es im Argument der Nichtabgeleitetheit jedoch nicht um die Zugehörigkeit mentaler Repräsentationen geht, benötige ich keine Antwort auf die Frage, wie diese Bedingung genau ausbuchstabiert werden sollte.
} 
von Photosynthese relevant ist, auch Informationen über die elektromagnetische Energie zu haben, die den Prozess der Photosynthese antreibt, ist es für die Erforschung kognitiver Prozesse wichtig, die Repräsentationen und Prozesse außerhalb des kognitiven Wesens zu kennen. Wollte man etwa ohne Wissen über die Existenz und Funktionsweise von Landkarten, Navigationsgeräten, Smartphones und Straßenschildern erklären, wie es möglich ist, dass Menschen ihren Weg zu weit entfernten Zielen finden, so würden sicherlich viele Phänomene nicht erklärt werden können. Empirische Kognitionswissenschaft muss folglich Umweltfaktoren als Inputs, Outputs oder auch Rückkopplungsschleifen in ihre Forschung mit einbeziehen. Dies gilt insbesondere, wenn eine starke Kopplung zwischen externen Faktoren und dem Geist besteht. Aus einer solchen Rolle für die Erforschung von Kognition alleine folgt jedoch noch lange nicht, dass Inputs, Outputs und Rückkopplungsschleifen selber mentale Zustände oder Prozesse sind.

\section{Nichtabgeleiteter Gehalt ist notwendig für mentale Repräsentationen}

Will man gegen die These des erweiterten Geistes argumentieren, so lässt sich dies in zwei Schritten tun. Zunächst wird ein notwendiges Kriterium dafür benötigt, dass ein Vehikel eine mentale Repräsentation eines bestimmten Wesens ist. Im zweiten Schritt muss gezeigt werden, dass eine Repräsentation dieses notwendige Kriterium nur dann erfüllt, wenn sie sich innerhalb der körperlichen Grenzen des Wesens befindet, zu dem sie gehört.

Ein Argument gegen die These des erweiterten Geistes, welches dem hier beschriebenem Schema entspricht, wurde von Frederick Adams, Kenneth Aizawa und Jerry Fodor aufgestellt. Im ersten Schritt ihrer Argumentation formulieren sie folgende notwendige Bedingung:

'Underived' content $[. .$.$] is the mark of the mental; underived content is what minds$ and only minds have. (Fodor 2008)

A first essential condition on the cognitive is that cognitive states must involve intrinsic, non-derived content. (Adams und Aizawa 2001, S. 48)

Adams, Aizawa und Fodor sehen das Haben nichtabgeleiteten Gehalts als notwendige Bedingung für mentale Repräsentationen an. Diese Prämisse ist der Beobachtung geschuldet, dass nicht-mentale Repräsentationen wie Straßenschilder, Diagramme, Baupläne und 
der geäußerte Satz „Das Museum of Modern Art ist in der 53. Straße“ ihre semantischen Gehalte auf andere Art und Weise haben, als unsere Wünsche, Überzeugungen und Absichten. Diese Grundidee wird von Adams und Aizawa wie folgt ausgedrückt:

Roughly speaking, the idea is that derived content arises from the way in which items are handled or treated by intentional agents. For the most part, things with derived content are assigned that content by intentional agents who already have thoughts with meaning. Underived content arises from conditions that do not require the independent or prior existence of other content, representations, or intentional agents. (Aizawa und Adams 2005, S. 662)

Diese Charakterisierung gibt zwar einen Einblick in die Unterscheidung, ist aber nicht präzise genug. Eine Präzisierung sollte insbesondere folgende Frage beantworten: Unter welchen Bedingungen gilt von einer Repräsentation, dass sie ihren Gehalt auf abgeleitete Art und Weise hat? Zunächst lässt sich festhalten, dass abgeleitete Gehalte immer von etwas anderem abgeleitet sind.

The first thing to observe about the derived/non-derived distinction is that it concerns the conditions in virtue of which an object bears a particular content. A thought might bear the content that the cat is hungry in virtue of satisfying some conditions on non-derived content, whereas a particular inscription on a piece of paper might bear that same content by satisfying some other conditions on derived content. To put the matter another way, there are two questions one might ask of a representation. The first is what content that representation bears; the second is what conditions make it the case that it bears that content. (Adams und Aizawa 2010, S. 582)

Das Haben eines Gehalts ist vergleichbar mit der Eigenschaft, eine Großmutter zu sein. Während Elfriede eine Großmutter aufgrund der Tatsache ist, dass ihre Tochter einen Sohn hat, hat eine Repräsentation letztlich Gehalt aufgrund von Tatsachen, die keine Gehalte betreffen. Die zugrunde liegende Annahme ist, dass das Haben eines bestimmten Gehalts keine primitive Eigenschaft ist, die zum grundlegenden Inventar der Welt gehört. Semantische Eigenschaften lassen sich (ebenso wie Verwandtschaftsbeziehungen) auf andere Eigenschaften oder Dinge zurückführen. Je nachdem, auf welche Entitäten sich der Gehalt einer Repräsentation zurückführen lässt, hat diese Repräsentation abgeleiteten oder nichtabgeleiteten Gehalt. Dabei legt Adams und Aizawas vorletztes Zitat zunächst nahe, dass Gehalte dann abgeleitet sind, wenn sie durch andere Repräsentationen oder deren 
Gehalte konstituiert sind. Dies ist jedoch nicht überzeugend, insbesondere da zwei zentrale Positionen in der Philosophie des Geistes davon ausgehen, dass die Gehaltseigenschaften mentaler Repräsentationen durch andere Repräsentationen und ihre Gehalte konstituiert sind - der semantische Holismus und die These von der Sprache des Geistes.

Das erste Problem für diese Charakterisierung von Abgeleitetheit ergibt sich aus der Position des semantischen Holismus in Bezug auf mentale Repräsentationen (u.a. vertreten von Brandom 2001 und Searle 2009):

Bedeutung ist eine relationale Eigenschaft. Etwas, das Bedeutung hat (eine Überzeugung), gibt es nur relativ auf anderes Bedeutungsvolles (andere Überzeugungen), das alles zusammen ein System von Bedeutungsvollem bildet (das System von Überzeugungen einer Person, eine Theorie, eine Sprache). (Esfeld|2002, S. 51, Hervorhebungen entfernt)

Besonders plausibel ist ein Holismus in Bezug auf theoretische Begriffe, die in wissenschaftlichen Theorien genutzt werden, um Entitäten zu bezeichnen, die nicht direkt beobachtet werden können. So gehören in der klassischen Mechanik der Physik, die sich mit der Bewegung von Körpern beschäftigt, die Begriffe „Kraft“, „Masse“, „kinetische Energie“ und „Impuls“ zu einem Begriffsnetz, das immer als Ganzes gelernt wird und deren Teile nicht unabhängig voneinander verstanden werden können (vgl. Block, 1998). Der semantische Holismus geht darüber hinaus davon aus, dass auch die Gehalte dieser Begriffe voneinander abhängen. Wäre ein Holismus in Bezug auf mentale Repräsentationen wahr, dann wäre der Gehalt dieser Repräsentationen immer durch den Gehalt anderer (mentaler) Repräsentationen bestimmt. Dennoch ist davon auszugehen, dass weder Fodor, noch Adams und Aizawa, obwohl sie alle keine holistischen Positionen vertreten, ihre These von der Nichtabgeleitetheit geistiger Zustände von der Wahrheit oder Falschheit eines semantischen Holismus in Bezug auf mentale Repräsentationen abhängig machen wollen.

Ein zweites Problem der Annahme, dass abgeleitete Gehalte dadurch charakterisiert sind, dass sie durch Repräsentationen oder deren Gehalte konstituiert sind, ergibt sich direkt aus der von Fodor (u. a. in 1987, S. 135-154) und Adams und Aizawa (2001, S. 49) vertretenen These von der Sprache des Geistes („language of thought“). Diese These beinhaltet die Annahme, dass sich die Gehalte komplexer mentaler Repräsentationen aus den Gehalten einfacher mentaler Repräsentationen ergeben können. Der Gehalt von zusammengesetzten Repräsentationen ergibt sich also konstitutiv aus den Teilrepräsentationen und ihren Gehalten. Ingas Überzeugung, dass Julia Romeo liebt, könnte somit ein neuronaler Zustand sein, für dessen Gehalt unter anderem der Gehalt zweier Teilzustände 
konstitutiv ist, von denen einer Julia und einer Romeo repräsentiert. Für zusammengesetzte Überzeugungen dieser Art gilt, dass andere Repräsentationen oder gar Überzeugungen konstitutiv dafür sind, dass sie ihren Gehalt haben. Dennoch wollen Fodor, Adams und Aizawa selbstverständlich nicht behaupten, dass zusammengesetzte mentale Repräsentationen abgeleiteten Gehalt haben. Dies würde schließlich der Annahme widersprechen, dass die Gehalte aller mentalen Repräsentationen nichtabgeleitet sind.

Um die Probleme abgeleiteten Gehalts mit Holismus und mit kompositionaler Semantik zu vermeiden, hilft es, einen Blick auf Adams, Aizawas und Fodors paradigmatische Beispiele für Repräsentationen mit abgeleitetem Gehalt zu werfen: Verkehrsampeln, Autotachos, Flaggen (vgl. Adams und Aizawa 2008, S. 32), klingelnde Telefone oder die Einträge in Ottos Notizbuch (vgl. Fodor 2008). Der Gehalt all dieser Repräsentationen hängt nicht von beliebigen anderen Repräsentationen oder ihren Gehalten ab. Vielmehr spielen in jedem Fall Absichten, Interpretationen oder Konventionen, die sich direkt oder indirekt auf die Repräsentation beziehen, eine Rolle.

Betrachten wir folgendes Beispiel. Tom fragt seine Freundin Sabine, wie der Bundestag in Bezug auf die Frage entschieden hat, ob gleichgeschlechtliche Paare heiraten dürfen. Was Sabine mit ihrer Geste repräsentiert, hängt plausiblerweise von der Absicht ab, die Sabine in Bezug auf ihre Geste hat. Grice zufolge wäre dies z. B. die Absicht, durch das Verbergen des Gesichtes bei Tom die Meinung hervorzurufen, dass der Bundestag die Ehe von gleichgeschlechtlichen Paaren abgelehnt hat - und zwar dadurch, dass Tom diese Absicht erkennt (vgl. Grice 1957, S. 385). Grices Theorie, welche insbesondere die Bedeutung improvisierter kommunikativer Zeichen gut erklären kann, ist also eine Theorie abgeleiteten Gehalts. Ebenso wäre eine Theorie, die den Gehalt eines Zeichens davon abhängig macht, wie dieses Zeichen interpretiert wird, eine Theorie abgeleiteten Gehalts. Gerade in Bezug auf Sprache ist jedoch anzunehmen, dass nicht nur Absichten oder Interpretationen, sondern auch Konventionen in Bezug auf bestimmte sprachliche Äußerungen (Adams und Aizawa 2001, S. 48) eine Rolle spielen. Konventionen sollen hier als soziale Konventionen (vgl. Rescorla 2011) und somit als absichtsgeleitete Verhaltensregularitäten verstanden werden, die aufgrund expliziter oder stillschweigender Übereinkunft eingehalten werden. Für Konventionen im hier gemeinten Sinne sind also bestimmte Absichten und Überzeugungen konstitutiv. Eine Theorie konventionaler Bedeutung, wie etwa die von David Lewis (1975, 2002), ist demnach eine Theorie abgeleiteten Gehalts. Ist eine Repräsentation abgeleitet, so gibt es eine Absicht, eine Interpretation oder eine Konvention, die sich auf diese Repräsentation bezieht und die konstitutiv dafür ist, dass sie einen bestimmten Gehalt hat. Ein solcher Bezug muss 
jedoch nicht direkt auf die Repräsentation gerichtet sein. Ein Thermometerstand von 82,53 ${ }^{\circ} \mathrm{C}$ hätte auch dann abgeleiteten Gehalt, wenn eine Absicht, Interpretation oder Konvention für seinen Gehalt konstitutiv ist, die sich auf Thermometerstände im allgemeinen und nicht nicht explizit diesen Thermometerstand bezieht. Zusammengefasst:

\section{Abgeleiteter Gehalt}

Eine Repräsentation $R$ hat den abgeleiteten Gehalt $G$ gdw. mindestens eine Absicht, Interpretation oder Konvention, die $R$ direkt oder indirekt zum Gegenstand hat, konstitutiv dafür ist, dass $R$ den Gehalt $G$ hat.

Die erste Prämisse von Fodors, Adams und Aizawas Argument gegen die These des erweiterten Geistes lässt sich nun wie folgt formulieren: Eine Repräsentation $R$ mit dem Gehalt $G$ ist nur dann eine mentale Repräsentation, wenn keine Absicht, Interpretation oder Konvention, die $R$ direkt oder indirekt zum Gegenstand hat, konstitutiv dafür ist, dass $R$ den Gehalt $G$ hat.

Diese Prämisse ist prima facie sehr plausibel. Die semantischen Eigenschaften kommunikativer Zeichen mögen auf entsprechenden Absichten, Interpretationen und Konventionen beruhen, wie etwa von Grice und Lewis behauptet. Für mentale Repräsentationen scheint diese Annahme jedoch zumindest kontraintuitiv zu sein. Aus der Definition abgeleiteten Gehalts folgt sogar, dass es mentale Repräsentationen (Absichten, Interpretationen oder die Überzeugungen und Absichten, die Konventionen zugrunde liegen) geben muss, deren Gehalt nicht abgeleitet ist, damit es überhaupt mentale Repräsentationen gibt. Denn für jede beliebige mentale Repräsentation gibt es zwei Möglichkeiten: Entweder ist ihr Gehalt abgeleitet oder er ist nichtabgeleitet. Ist der Gehalt dieser Repräsentation nicht abgeleitet, so gibt es mentale Repräsentationen mit nichtabgeleitetem Gehalt. Ist ihr Gehalt hingegen abgeleitet, so muss es eine weitere mentale Repräsentation geben, die sich auf die erste mentale Repräsentation bezieht. In diesem Fall stellt sich die Frage nach der Abgeleitetheit oder Nichtabgeleitetheit dieser zweiten mentalen Repräsentation und so fort. Wird dieser Zirkel nicht an einer Stelle durch eine Absicht, Interpretation oder Konvention mit nichtabgeleitetem Gehalt durchbrochen, ist nicht zu sehen, wie Gehalte überhaupt in die Welt kommen können (vgl. Adams und Aizawa 2001, S. 49; 2008, S. 33f.).

Einige mentale Repräsentationen haben also fraglos nichtabgeleiteten Gehalt. Ein Argument dafür, dass die semantischen Eigenschaften aller mentalen Repräsentationen nichtabgeleitet sind, geben Adams, Aizawa und Fodor nicht und auch mir ist kein solches Argument bekannt. Allerdings lassen sich zu dieser Prämisse auch keine offensichtlichen Gegenbeispie- 
le finden. Die erste Prämisse des Arguments der Nichtabgeleitetheit kann demnach, wenn schon nicht als gesichert, dann doch als sehr plausibel betrachtet werden.

\section{Nichtabgeleiteter Gehalt nur innerhalb des Körpers}

Auf Grundlage dieser ersten Prämisse argumentieren Adams, Aizawa und Fodor im zweiten Schritt gegen externe mentale Zustände. Dabei vertritt Fodor die moderate These, dass typische Beispiele für externe mentale Zustände, wie etwa die Einträge im Notizbuch Ottos, keinen nichtabgeleiteten Gehalt besitzen und daher auch keine mentalen Zustände sind. Adams und Aizawa (vgl. auch 2008, S. 55) hingegen behaupten mehr:

Whatever is responsible for non-derived representations seems to find a place only in brains. (Adams und Aizawa 2001, S. 63)

Da sich das Gehirn im Körper befindet, gäbe es folglich nur interne Repräsentationen mit nichtabgeleiteten Gehalten. Wäre zudem nichtabgeleiteter Gehalt eine notwendige Bedingung für mentale Repräsentationen, würde folgen, dass mentale Repräsentationen nur in zentralen Nervensystemen zu finden seien. Das Argument der Nichtabgeleitetheit gegen die These des erweiterten Geistes hat somit folgende einfache Struktur:

\section{Das Argument der Nichtabgeleitetheit}

Prämisse 1: Eine Repräsentation ist nur dann eine mentale Repräsentation, wenn ihr Gehalt nicht abgeleitet ist.

Prämisse 2: Es gibt keine externen Repräsentationen, deren Gehalt nicht abgeleitet ist.

Konklusion: Es gibt keine externen mentalen Repräsentationen.

Dieses Argument ist fraglos gültig. Wären auch seine Prämissen wahr, gäbe es keine geistigen Repräsentation außerhalb des zentralen Nervensystems. Die These des erweiterten Geistes wäre somit falsch. Otto hätte keine Überzeugungen, Wünschen und Absichten in seinem Notizbuch und auch unsere Smartphones wären keine Erweiterungen unseres Gedächtnisses. Ich werde im Folgenden Kritik an diesem Argument üben. Dabei werde ich nicht seine erste Prämisse und somit seine theoretische Grundlage attackieren, sondern die zweite, empirische Prämisse angreifen. Repräsentationen mit nichtabgeleiteten Gehalten finden sich, so werde ich zeigen, durchaus auch außerhalb kognitiver Wesen. 


\section{Die Quellen der Erfüllungsstandards}

Ich werde dafür argumentieren, dass es gute Kandidaten für externe mentale Repräsentationen gibt, die die von Adams, Aizawa und Fodor aufgestellte Anforderung erfüllen. Ein solches Argument setzt eine Theorie nichtabgeleiteten Gehalts voraus. Ich werde mich für meine weitere Argumentation auf die Teleosemantik stützen. Dies hat zwei Gründe. Zum einen beziehen sich Adams und Aizawa (2001, S. 48, 2008, S. 36f.) in ihrer Erklärung dessen, was sie mit nichtabgeleitetem Gehalt meinen, unter anderem auf die teleosemantischen Theorien von Millikan (1984) und Dretske (1988). Zum anderen handelt es sich bei der Teleosemantik um die aussichtsreichste und meistdiskutierte Theorie nichtabgeleiteten Gehalts. Um den teleosemantischen Ansatz plausibel zu machen, werde ich zeigen, dass die Erfüllungsstandards im Fall nichtabgeleiteten Gehalts am besten mit Hilfe natürlicher Funktionen erklärt werden können. Doch zunächst werde ich herausarbeiten, wie Absichten, Interpretationen und Konventionen die Erfüllungsstandards von Repräsentationen mit abgeleitetem Gehalt erklären können.

Absichten, Interpretationen und Konventionen machen den Unterschied zwischen Dingen mit natürlicher Bedeutung und Repräsentationen mit abgeleitetem Gehalt aus, indem sie die Erfüllungsstandards letzterer konstituieren. Bleiben wir etwa bei der Grice'schen Theorie, könnten wir sagen, dass der Satz „Der Patient hat Masern“ deshalb bedeutet, dass der Patient Masern hat, weil mit der Äußerung dieses Satzes die Absicht verbunden war, beim Hörer die Meinung hervorzurufen, dass der Patient Masern hat. Sätze können demnach auch dann bedeuten, dass der Patient Masern hat, wenn der Patient tatsächlich keine Masern hat, weil diese Sätze mit der Absicht verbunden waren, beim Hörer die Meinung hervorzurufen, dass der Patient Masern hat; diese Absicht kann auch vorliegen, wenn der Patient tatsächlich keine Masern hat. In diesem Fall liegt eine Fehlrepräsentation vor. Auch die Spezifität von abgeleiteten Gehalten lässt sich mit Hilfe von Absichten erklären. Sätze können bedeuten, dass der Patient Masern hat, ohne gleichzeitig zu bedeuten, dass der Patient eine Krankheit hat oder dass der Patient sich bei seiner Schwester angesteckt hat, da diese Sätze nicht mit der Absicht verbunden waren, beim Hörer die Meinung hervorzurufen, dass der Patient eine Krankheit hat oder dass der Patient sich bei seiner Schwester angesteckt hat; wohl aber, dass er Masern hat. Auf ähnliche Art und Weise lassen sich mit Hilfe von Interpretationen oder Konventionen die Erfüllungsstandards von Dingen mit abgeleitetem Gehalt erklären. Entscheidend für diese Erklärungen ist, dass Überzeugungen, Interpretationen, Absichten und somit auch Konventionen Erfüllungsstandards unterliegen. Es handelt sich bei ihnen um mentale, nichtabgeleitete Repräsentationen mit 
nicht-natürlicher Bedeutung oder im Falle von Konventionen um komplexe Eigenschaften, die unter anderem durch solche Repräsentationen konstituiert sind.

Aber was erklärt die Nicht-Faktivität und die Spezifität nichtabgeleiteten Gehalts? Die wohl aussichtsreichste Antwort auf diese Frage liefert der teleologische Ansatz, der auch unter den Bezeichnungen „Teleosemantik“ oder „Biosemantik“ bekannt ist (vgl. u.a. Millikan 1984; Papineau 1984; Dretske 1988; Sterelny 1991; Neander 1995). Die Teleosemantik nimmt Bezug auf den Begriff der natürlichen Funktion, der insbesondere in der Biologie und Medizin eine wichtige Rolle spielt. Etwas hat eine Funktion in diesem Sinne, wenn es dazu da ist, etwas bestimmtes zu tun, oder anders formuliert, wenn es etwas bestimmtes tun "soll". Vertreter der Teleosemantik gehen meist von einem ätiologischen Verständnis natürlicher Funktionen aus. Dieses ist evolutionsbiologisch inspiriert und impliziert, dass etwas genau dann die Funktion hat, zu $\varphi$-en, wenn es dafür selektiert wurde, zu $\varphi$-en (Wright 1973; Millikan 1984; Neander 1991). Das Herz hat dieser Theorie zufolge genau deshalb die Funktion, Blut zu pumpen, weil Herzen in der Vergangenheit Blut gepumpt haben und weil Herzen genau aus diesem Grund in der Vergangenheit selektiert wurden.5

Prinzipiell ist gut verständlich, wie die Erfüllungsstandards von Dingen mit nichtabgeleitetem Gehalt nur unter Rückgriff auf einen solchen Funktionsbegriff und natürliche Bedeutung, verstanden im Sinne von Korrelations- oder Kausalbeziehungen, erklärt werden können. Der Grund hierfür ist einfach: Auch Dinge mit natürlichen Funktionen unterliegen Erfüllungsstandards. Zum einen ist es ohne Weiteres möglich, dass etwas eine bestimmte Aufgabe erfüllen soll, obwohl es diese Aufgabe nicht erfüllt. So wurden Herzen etwa dafür selektiert, Blut zu pumpen. Die ätiologische Funktion eines jeden Herzens ist das Pumpen von Blut. Dennoch pumpt ein Herz, das akut an Herzkammerflimmern leidet, kein Blut. Ein solches Herz hat eine Fehlfunktion. Eine natürliche Funktion zu haben, ist also eine nicht-faktive Eigenschaft. Teleosemantische Theorien können die Tatsache, dass es Fehlfunktionen gibt, nutzen, um Fehlrepräsentationen zu erklären (Dretske 1988, 1990). So ist etwas, Dretske zufolge, eine Repräsentation mit dem Gehalt, dass eine Fliege in der Nähe

\footnotetext{
${ }^{5}$ Doch erlaubt ein solcher Funktionsbegriff nicht nur den Erwerb von Funktionen in der Phylogenese eines Organismus. Auch ontogenetische Lern- und Kondititionierungsprozesse stellen Selektionen im relevanten Sinne dar und können somit dafür sorgen, dass bestimmte Merkmale eines Organismus Funktionen erwerben (vgl. Dretske 1988, S. 99 - 107; Millikan 2004, Kapitel 1; Macdonald und Papineau 2006, S. 14ff.).
} 
ist, wenn es die Funktion hat 5 , anzuzeigen, dass eine Fliege in der Nähe ist.7

Da etwas aber auch eine Funktion haben kann, ohne sie zu erfüllen, kann etwas die Funktion haben, anzuzeigen, dass eine Fliege in der Nähe ist, ohne dass es die Anwesenheit einer Fliege in der Nähe anzeigt - z.B. deshalb, weil keine Fliege in der Nähe ist. Das ist es, was wir meinen, wenn wir von Fehlrepräsentationen sprechen. Die teleosemantische Theorie liefert also eine plausible und elegante Antwort auf die Frage, wie Fehlrepräsentationen möglich sind, ohne dabei auf andere Repräsentationen zurückgreifen zu müssen. Die Nicht-Faktivität nichtabgeleiteten Gehalts lässt sich auf die Nicht-Faktivität natürlicher Funktionen zurückführen.

Gleiches ist für die Spezifität nichtabgeleiteten Gehalts möglich. Etwas kann die natürliche Funktion haben, einen bestimmten Zustand herbeizuführen, ohne die Funktion zu haben, einen anderen Zustand herbeizuführen, auch wenn beide Zustände immer zusammen bestehen oder einander verursachen. Auch wenn Herzen dafür selektiert wurden, Blut zu pumpen, wurden sie nicht dafür selektiert, alle sich im Blut befindlichen Viren im Körper zu verteilen, auch wenn natürlich beide Effekte des Herzens immer zusammenhängen. Die Eigenschaft, eine Funktion zu haben, ist also spezifisch. Die Spezifität natürlicher Funktionen ermöglicht die Erklärung des spezifischen Gehalts mentaler Repräsentationen. Man kann sich in einem Gehirnzustand befinden, der in einem Lernprozess dafür selektiert wurde, anzuzeigen, dass der Patient Masern hat, ohne dass dieser Gehirnzustand dafür selektiert wurde, anzuzeigen, dass er sich bei einem anderen Menschen per Tröpfeninfektion mit dem Masernvirus infiziert hat oder dass er die nächsten Tage nicht arbeiten kann. Diese Tatsache erklärt, warum es möglich ist, zu glauben, dass der Patient Masern hat, ohne gleichzeitig zu glauben, dass er sich bei einem anderen Menschen per Tröpfeninfektion mit dem Masernvirus infiziert hat oder zu glauben, dass der Patient die nächsten Tage nicht arbeiten kann.

Prima facie ist es also plausibel, anzunehmen, dass die Erfüllungsstandards von Repräsentationen mit Hilfe eines ätiologischen Funktionsbegriffs erklärt werden können. Eini-

\footnotetext{
${ }^{6}$ Aus Platzgründen werde ich in diesem Aufsatz nur diese einfache Version der Teleosemantik besprechen, der zufolge die Funktion der Repräsentation ihren Gehalt bestimmt. Dem gegenüber stehen so genannte Produzenten- bzw. Konsumententheorien, denen zufolge der Gehalt einer Repräsentation von der Funktion des Systems, das die Repräsentation produziert bzw. nutzt, bestimmt wird. Produzententheorien werden unter anderem von Dretske (1995) und Neander (2012), Konsumententheorien beispielsweise von Millikan (1989) und Stegmann (2009) vertreten. Meine weitere Argumentation lässt sich problemlos auch auf diese Versionen der Teleosemantik übertragen.

${ }^{7}$ Dabei versteht Dretske (1988, S. 55) unter der Anzeigebeziehung das, was Grice als "Bedeutung $n$ " versteht. Diese Beziehung buchstabiert er (1981) im Sinne einer komplexen Korrelationsbeziehung aus.
} 
ge problematische Aspekte der Teleosemantik können an dieser Stelle nicht weiter thematisiert werden. Hierzu gehört etwa die Möglichkeit nichtabgeleiteter Gehalte ohne Evolutionsoder Lerngeschichte (vgl. Braddon-Mitchell und Jackson 1997), die Frage nach unserer Rechtfertigung beim Zuschreiben nichtabgeleiteter Gehalte (vgl. Jackson 2006) oder die Frage, ob teleosemantische Theorien plausible Verhaltenserklärungen ermöglichen, die auf Repräsentationen und ihre Gehalte Bezug nehmen (vgl. Block 1989, S. 53f.). Aber keine Theorie ist ohne Probleme und mir scheint der teleosemantische Ansatz der vielversprechendste zu sein.

\section{$6 \quad$ Nichtabgeleiteter Gehalt außerhalb des Körpers}

Im diesem Abschnitt werde ich anhand eines Beispiels zeigen, dass es auch außerhalb der körperlichen Grenzen von Tieren Repräsentationen mit nichtabgeleitetem Gehalt gibt. Es gibt Fälle, in denen Dinge in der Umwelt von Tieren natürliche Anzeigefunktionen besitzen.

Der ätiologische Funktionsbegriff ist nicht auf Organe von Tieren oder gar auf Teile des zentralen Nervensystems beschränkt. Auch des Verhalten von Tieren und durch sie produzierte Dinge können die Funktion haben, bestimmte Umweltbedingungen anzuzeigen. Beispielsweise führen Westliche Honigbienen (Apis mellifera) einen so genannten Schwänzeltanz aus, wenn sie eine Nahrungsquelle in größerer Entfernung zu ihrem Bienenstock gefunden haben. Dieser Tanz entspricht etwa der Form eines großen Phi $(\Phi)$, wobei die mittlere Linie, begleitet von heftigem seitlichem Vibrieren des Hinterleibs, immer in eine Richtung bestritten wird (Schwänzellauf). Je langsamer der Rhythmus des Tanzes, desto größer ist die Entfernung der gefundenen Nahrungsquelle. Die Richtung des Schwänzellaufs zeigt hingegen die Richtung der Futterquelle in Bezug zum Sonnenstand an (vgl. von Frisch 1946). Da dieser Schwänzeltanz dafür selektiert wurde, Informationen über die Richtung und die Entfernung einer Nahrungsquelle zu tragen, stellt jeder Tanz dieser Art eine Repräsentation mit nichtabgeleitetem Gehalt dar. Weitere Beispiele für Repräsentationen mit nichtabgeleitetem Gehalt sind unter anderem die evolutionär entstandenen Warnsignale von Bibern und Meerkatzen, die nahende Gefahr repräsentieren. Alle diese Repräsentationen befinden sich außerhalb der körperlichen Grenzen kognitiver Wesen. Die zweite Prämisse des Arguments der Nichtabgeleitetheit ist also schon durch diese Beispiele widerlegt.

Man könnte jedoch folgendes entgegnen: Bei den genannten Beispielen haben wir es zwar mit externen Repräsentationen mit nichtabgeleitetem Gehalt zu tun, dennoch han- 
delt es sich klarerweise nicht um Fälle kognitiver Erweiterung, sondern um Kommunikation. Das Argument der Nichtabgeleitetheit sollte somit modifiziert werden. Mentale Repräsentationen haben nicht nur nichtabgeleiteten Gehalt, sondern dienen außerdem nicht der Kommunikation.

Es stellt sich also die Frage, wie sich zwischen mentalen Repräsentationen und kommunikativen Repräsentationen unterscheiden lässt. Ein möglicher Ansatz zur Beantwortung dieser Frage liefert die in der Teleosemantik übliche Unterscheidung zwischen dem Produzenten und dem Nutzer einer Repräsentation (vgl. Millikan 1989, S. 285). Kognitive Repräsentationen werden im Gegensatz zu kommunikativen Repräsentationen von Systemteilen produziert und genutzt, die zu demselben Organismus gehören. Dies ist für die Tänze der Honigbiene und auch für die Warnsignale von Bibern und Meerkatzen nicht der Fall. Daher handelt es sich bei ihnen nicht um kognitive Repräsentationen.

Ein klares Beispiel für externe Repräsentationen, bei denen Produzent und Nutzer zum selben Organismus gehören, stellen die Duftmarkierungen dar, die einige Mitglieder der Familie der Hunde (Canidae) während der Nahrungssuche anlegen und nutzen. Dieses Verhalten wurde zuerst von J. David Henry (1977) bei Rotfüchsen (Vulpes vulpes) und dann von Fred H. Harrington (1981 und 1982) bei Wölfen (Canis lupus) und Kojoten (Canis latrans) beobachtet:

In the red fox, urine-marking occurs frequently during foraging (Henry, 1977). Typically, after a fox empties a cache, the empty hole is urine-marked. This olfactory cue may later inform that same fox (or another) that no edible food remains, although food odors might still linger. Thus the fox need not waste additional time digging up the site. Such a ,book-keeping" system should increase foraging efficiency, permitting the fox to concentrate its efforts on sites with the greatest potential for payoff. Indeed, Henry demonstrated that foxes spent only one-fifth as much time investigating urine-marked exploited caches, as they did investigating empty but un-marked caches. (Harrington 1981, S. 280)

Eine naheliegende evolutionäre Erklärung für dieses Verhalten setzt voraus, dass die Vorfahren der heutigen Füchse, Wölfe und Kojoten, die dieses Verhalten an den Tag legten, weniger Zeit damit verbrachten, nicht essbare Nahrungsreste zu suchen. Aus diesem Grund hatten sie größeren Erfolg bei der Nahrungssuche und konnten sich somit gegen andere Lebewesen, die dieses Verhalten nicht aufwiesen, durchsetzen. Wenn diese Erklärung korrekt ist, haben die Urinmarkierungen von Füchsen, Wölfen und Kojoten die ätiologische 
Funktion, die Information zu tragen, dass sich an der markierten Stelle keine Nahrung befindet. Sie repräsentieren also die Abwesenheit von Nahrung und sind inkorrekt, wenn sich an der markierten Stelle Essbares befindet. Urinmarkierungen repräsentieren spezifisch die Abwesenheit von Nahrung und nicht etwa die Abwesenheit ehemals lebendiger Zellen, auch wenn die Nahrung von Füchsen, Kojoten und Wölfen üblicherweise aus ehemals lebendigen Zellen besteht.

Die Gehalte der Urinmarkierungen von Füchsen, Wölfen und Kojoten haben also nichtabgeleiteten Gehalt und dienen nicht der Kommunikation. Die Duftmarkierungen befinden sich jedoch klarerweise außerhalb der körperlichen Grenzen kognitiver Wesen. Es gibt demnach externe, nichtkommunikative Repräsentationen mit nichtabgeleiteten Gehalten. Folglich ist auch das modifizierte Argument der Nichtabgeleitetheit nicht haltbar. Duftmarkierungen werden jedoch nicht nur von Mitgliedern der Familie der Hunde genutzt, um ihre Nahrungssuche zu optimieren. Honigbienen (Apis mellifera) und Hummeln (Bombus) weisen ein ganz ähnliches Verhalten auf, indem sie Blumen, die gerade abgeerntet wurden oder besonders ertragreich sind, mit bestimmten Düften markieren (vgl. Stout und Goulson 2001). Andere mögliche Beispiele stellen kleine Hügel dar, die balzende, männliche Winkerkrabben (Uca terpsichores) in die Nähe ihrer Höhlen errichten und die ihnen unter anderem als visueller Reiz dienen, der ihnen hilft, bei Gefahr in ihre Höhlen zurückzukehren (vgl. Ribeiro u. a. 2006) ${ }^{8}$

Der Teleosemantik zufolge gibt es externe Repräsentationen mit nichtabgeleitetem Gehalt. Natürlich ließe sich die Teleosemantik und somit die meiner Argumentation zugrundeliegende Theorie mentalen Gehalts verwerfen. Dieser Schritt hätte jedoch einen starken ad hoc-Charakter und wird auch von Adams und Aizawa nicht vollzogen.

\section{Asymmetrische Abhängigkeit als Rettung?}

Fodor auf der anderen Seite würde sicherlich die teleosemantische Theorie ablehnen. Ohne eine Theorie nichtabgeleiteten Gehalts lässt sich das Argument der Nichtabgeleitetheit jedoch nicht untersuchen. Ich will an dieser Stelle kurz Fodors eigene Theorie der asymmetrischen Abhängigkeit (vgl. Fodor 1987, 1990) als Alternative zur Teleosemantik betrachten.

Fodor geht zunächst davon aus, dass der Gehalt einer Repräsentation durch eine einfache Kausalbeziehung konstituiert ist. Eine Kuh-Repräsentation repräsentiert die Anwe-

\footnotetext{
${ }^{8}$ Weitere Beispiele für evolutionär entstandene "Autokommunikation" im Tiereich finden sich in Schaedelin und Taborsky (2009, S. 289).
} 
senheit von Kühen, weil sie von Kühen verursacht wird. Wie wir jedoch gesehen haben, sind einfache Kausalbeziehungen faktiv und nicht spezifisch, während der Gehalt einer Repräsentation immer nicht-faktiv und spezifisch ist. Um diese Eigenschaften einzufangen, unterscheidet Fodor zwischen primärer und sekundärer kausaler Abhängigkeit. Sekundäre Abhängigkeit liegt vor, wenn etwa eine Kuh-Repräsentation durch ein Pferd im Dunkeln verursacht wird. Denn in diesem Fall gilt: Wenn Kühe nicht Kuh-Repräsentationen verursachen würden, würden auch keine Pferde im Dunkeln Kuh-Repräsentationen verursachen. Kuh-Repräsentationen sind hingegen primär kausal abhängig von von Kühen, weil für Kühe nicht gilt, dass sie Kuh-Repräsentationen nur deshalb verursachen, weil Pferde im Dunkeln, Kuhattrappen oder andere Dinge Kuh-Repräsentationen verursachen. Aus diesem Grund repräsentieren Kuh-Repräsentationen, Fodor zufolge, Kühe und nicht Pferde im Dunkeln, Kuhattrappen oder andere Dinge.

Abgesehen von der Frage, ob Fodors Theorie wirklich eine adäquate Theorie nichtabgeleiteten Gehalts darstellt, ist nicht zu sehen, wie sie externe nichtabgeleitete Repräsentationen ausschließen kann. Es ist zwar auch so, dass eingepacktes oder geruchloses Futter Duftmarkierungen verursacht. Doch wenn im Fall von Kühen und Pferden eine asymmetrische Abhängigkeit vorliegt, dann auch hier: Duftmarkierungen wären nicht kausal abhängig von Situationen mit eingepacktem oder geruchlosem Futter, wenn sie nicht kausal von Situationen ohne essbares Futter abhängig wären. Duftmarkierungen sind also primär kausal abhängig von Situationen, an denen kein essbareres Futter mehr vorhanden ist. Es ist nicht zu sehen, wie Fodor ausschließen kann, dass Duftmarkierungen die Abwesenheit von essbarem Futter als nichtabgeleiteten Gehalt haben.

\section{Fazit}

Adams, Aizawa und Fodor formulieren mit dem Argument der Nichtabgeleitetheit ein prima facie sehr plausibles Argument gegen die These des erweiterten Geistes. Diese Plausibilität lässt sich allerdings nicht aufrecht erhalten. Grund hierfür ist nicht das Fehlschlagen der zugrundeliegenden theoretischen Annahme, der zufolge mentale Gehalte immer nichtabgeleitet sind, sondern das Fehlschlagen der empirischen Hypothese, dass externe Repräsentationen nur abgeleiteten Gehalt haben.

Schon Bienentänze und Warnsignale von Bibern und Meerkatzen stellen hier Gegenbeispiele dar. Auch mit der zusätzlichen Bedingung, dass mentale Repräsentationen immer vom gleichen Organismus produziert und genutzt werden, lässt sich das Argument nicht 
halten. Die Duftmarkierungen von Füchsen, Wölfen und Kojoten und eine Reihe weiterer Beispiele erfüllen auch diese Bedingung. Dies folgt zumindest aus der Teleosemantik, der aussichtsreichsten und meistdiskutierten Theorie mentalen Gehalts, und, wie ich gezeigt habe, vermutlich sogar aus Fodors eigener Theorie mentalen Gehalts. In Anbetracht dieser Tatsache ist zweifelhaft, ob andere Theorien nichtabgeleiteten Gehalts das von Adams, Aizawa und Fodor gewünschte Ergebnis liefern. Dies führt zu einer klaren Verschiebung der Beweislast. Die Vertreter des Arguments der Nichtabgeleitetheit können sich nicht auf die Annahme stützen, der Gehalt externer Repräsentationen sei immer abgeleitet, auch wenn einzelne Beispiele vermeintlicher mentaler Erweiterung, wie etwa der von Otto, durch das Argument der Nichtabgeleitetheit ausgeschlossen werden können.

Habe ich damit gezeigt, dass die These des erweiterten Geistes wahr ist? Nicht notwendigerweise. Dies hätte ich nur, wenn das Haben nichtabgeleiteten Gehalts und die Bedingung, vom selben Organismus produziert und genutzt zu werden, hinreichend dafür wäre, eine mentale Repräsentation dieses Organismus zu sein. Die Haltbarkeit dieser These war jedoch nicht Thema dieser Arbeit. Mein Ziel war es lediglich, zu zeigen, dass das Argument der Nichtabgeleitetheit kein überzeugendes Argument gegen die These des erweiterten Geistes darstellt.

\section{Literatur}

[Adams und Aizawa 2001] Adams, Frederick R. ; Aizawa, Kenneth: The bounds of cognition. In: Philosophical Psychology 14 (2001), Nr. 1, S. 43-64

[Adams und Aizawa 2008] Adams, Frederick R. ; Aizawa, Kenneth: The bounds of cognition. Malden, Mass. [u.a.] : Blackwell, 2008

[Adams und Aizawa 2010] Adams, Frederick R. ; AizawA, Kenneth: The value of cognitivism in thinking about extended cognition. In: Phenomenology and the Cognitive Sciences 9 (2010), Nr. 4, S. 579-603

[Aizawa 2010] AIzAwa, Kenneth: The coupling-constitution fallacy revisited. In: Cognitive Systems Research 11 (2010), Nr. 4, S. 332-342

[Aizawa und Adams 2005] Aizawa, Kenneth ; Adams, Frederick R.: Defending NonDerived Content. In: Philosophical Psychology 18 (2005), Nr. 6, S. 661-669 
[Block 1989] Block, Ned: Can the Mind Change the World? In: Meaning and Method: Essays in Honor of Hilary Putnam. Cambridge University Press, 1989, S. 137-170

[Block 1998] Block, Ned: Holism: mental and semantic. In: CrAIG, Edward (Hrsg.): Routledge Encyclopedia of Philosophy. London : Routledge, 1998

[Braddon-Mitchell und Jackson 1997] Braddon-Mitchell, David ; JACKson, Frank: The Teleological Theory of Content. In: Australasian Journal of Philosophy 75 (1997), Nr. 4, S. 474-89

[Brandom 2001] Brandom, Robert: Begründen und Begreifen: Eine Einführung in den Inferentialismus. Frankfurt am Main : Suhrkamp, 2001

[Clark und Chalmers 1998] Clark, Andy ; Chalmers, David J.: The Extended Mind. In: Analysis 58 (1998), Nr. 1, S. 7-19

[Dretske 1981] Dretske, Fred: Knowledge and the flow of information. Oxford : Blackwell, 1981

[Dretske 1988] Dretske, Fred: Explaining behavior. Cambridge, Mass. [u.a.] : MIT Press, 1988

[Dretske 1990] Dretske, Fred: Misrepresentation. In: LyCAN, William G. (Hrsg.): Mind and Cognition: A Reader. MIT Press, 1990, S. 129-143

[Dretske 1995] Dretske, Fred: Naturalizing the Mind. The MIT Press, 1995

[Esfeld 2002] Esfeld, Michael: Was besagt semantischer Holismus? Zwei Möglichkeiten der Konzeptualisierung. In: Bertram, Georg W. (Hrsg.) ; Liptow, Jasper (Hrsg.): Holismus in der Philosophie: ein zentrales Motiv der Gegenwartsphilosophie. Weilerswist: Velbrück Wissenschaft, 2002, S. 41-58

[Fodor 1987] Fodor, Jerry A.: Psychosemantics. MIT Press, 1987 (Explorations in cognitive science ; 2)

[Fodor 1990] Fodor, Jerry A.: A theory of content and other essays. Cambridge, Mass. [u.a.] : MIT Press, 1990

[Fodor 2008] Fodor, Jerry A.: Where is my mind? In: London Review of Books 31 (2008), Nr. 3 
[von Frisch 1946] Frisch, Karl von: Die Tänze der Bienen. In: Österreichische Zoologische Zeitschrift 1 (1946), S. 1-48

[Grice 1957] Grice, H. P.: Meaning. In: The Philosophical Review 66 (1957), Nr. 3, S. $377-388$

[Harrington 1981] Harrington, Fred H.: Urine-Marking and Caching Behavior in the Wolf. In: Behaviour 76 (1981), Nr. 3/4, S. S. 280-288

[Harrington 1982] HARRIngton, Fred H.: Urine marking at food and caches in captive coyotes. In: Canadian Journal of Zoology 60 (1982), Nr. 5, S. 776-782

[Henry 1977] HenRy, J. David: The Use of Urine Marking in the Scavenging Behavior of the Red Fox (Vulpes vulpes). In: Behaviour 61 (1977), Nr. 1/2, S. S. 82-106

[Jackson 2006] JACKSON, Frank: The Epistemological Objection to Opaque Teleological Theories of Content. In: Macdonald, Graham (Hrsg.) ; Papineau, David (Hrsg.): Teleosemantics: new philosophical essays. Oxford University Press, 2006, S. 85-99

[Lewis 1975] Lewis, David: Languages and Language. In: Minnesota Studies in the Philosophy of Science Bd. 7. University of Minnesota Press, 1975, S. 3-35

[Lewis 2002] Lewis, David: Convention: A Philosophical Study. Wiley, 2002

[Macdonald und Papineau 2006] Macdonald, Graham ; Papineau, David: Introduction: Prospects and Problems for Teleosemantics. In: Teleosemantics: New Philosophical Essays. Oxford University Press, 2006, S. 1-22

[Millikan 1984] Millikan, Ruth Garrett: Language, Thought and Other Biological Categories. MIT Press, 1984

[Millikan 1989] Millikan, Ruth Garrett: Biosemantics. In: The Journal of Philosophy 86 (1989), Nr. 6, S. 281-297

[Millikan 2004] Millikan, Ruth Garrett: Varieties of meaning. Cambridge : MIT Press, 2004 (The Jean Nicod lectures 2002)

[Neander 1991] NeAnder, Karen: The teleological notion of ,function‘. In: Australasian Journal of Philosophy 69 (1991), Nr. 4, S. 454-468 
[Neander 1995] NeAnder, Karen: Misrepresenting \& malfunctioning. In: Philosophical Studies 79 (1995), S. 109-141

[Neander 2012] NeAnder, Karen: Toward an Informational Teleosemantics. In: KingsBURY, Justine (Hrsg.) ; RYder, Dan (Hrsg.) ; Williford, Kenneth (Hrsg.): Millikan and her Critics. Oxford : Blackwell, 2012, Kap. 1, S. 21-36

[Papineau 1984] Papineau, David: Representation and Explanation. In: Philosophy of Science 51 (1984), Nr. December, S. 550-572

[Putnam 1979] Putnam, Hillary: The meaning of 'meaning'. In: Putnam, Hilary (Hrsg.): Mind, Language and Reality. Cambridge : Cambridge University Press, 1979, S. $215-271$

[Rescorla 2011] Rescorla, Michael: Convention. In: N. Zalta, Edward (Hrsg.): The Stanford Encyclopedia of Philosophy. Spring 2011. 2011

[Ribeiro u. a. 2006] Ribeiro, Pablo D. ; Christy, John H. ; Rissanen, Rebecca J. ; KIm, Tae W.: Males Are Attracted by Their Own Courtship Signals. In: Behavioral Ecology and Sociobiology 61 (2006), Nr. 1, S. S. 81-89

[Rowlands 2009] Rowlands, Mark: Extended Cognition and the Mark of the Cognitive. In: Philosophical Psychology 22 (2009), Nr. 1, S. 1-19

[Rowlands 2010] Rowlands, Mark: The New Science of the Mind: From Extended Mind to Embodied Phenomenology. MIT Press, 2010

[Schaedelin und Taborsky 2009] Schaedelin, Franziska C. ; TABorsky, Michael: Extended phenotypes as signals. In: Biological Reviews 84 (2009), Nr. 2, S. 293-313

[Searle 2009] SEARLE, John Rogers: Intentionalität. Suhrkamp, 2009 (SuhrkampTaschenbuch-Wissenschaft 956)

[Stegmann 2009] Stegmann, Ulrich E.: A Consumer-Based Teleosemantics for Animal Signals. In: Philosophy of Science 76 (2009), Nr. 5, S. 864-875

[Sterelny 1991] SteRELNy, Kim: The representational theory of mind - an introduction. Oxford : Blackwell, 1991 
[Stout und Goulson 2001] Stout, Jane Catherine ; Goulson, Dave: The use of conspecific and interspecific scent marks by foraging bumblebees and honey- bees. In: Animal Behaviour 62 (2001), S. 183-189

[Wright 1973] Wright, Larry: Functions. In: Philosophical Review 82 (1973), Nr. 2, S. $139-168$ 